\title{
Neues zur Einteilung und Therapie von Meningeomen
}

Corinna Seliger, Wolfgang Wick

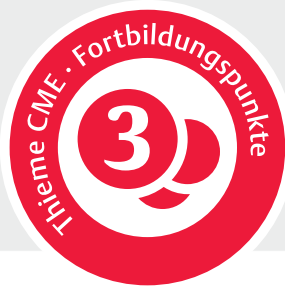

Meningeome gehören zu den häufigsten Hirntumoren bei Erwachsenen. Während die meisten Meningeome gutartig sind, treten seltener auch atypische oder anaplastische Meningeome auf. Der Goldstandard zur Behandlung von Meningeomen ist die Tumorresektion, die ggf. postoperativ um eine Strahlentherapie ergänzt wird. Relevante Systemtherapien gibt es nicht. Aktuell werden verschiedene molekular zielgerichtete Substanzen zur Behandlung von lokal nicht mehr therapierbaren Meningeomen untersucht.

\section{FALLBEISPIEL}

Fall 1

Ein 69-jähriger Patient stellte sich in der Notaufnahme vor, da er während eines Spaziergangs das Gefühl hatte, sein linkes Bein nicht mehr richtig koordinieren zu können. Er sei deshalb auch gestürzt, habe das Bewusstsein aber nicht verloren. Bei Eintreffen in der Notaufnahme hatte sich die Symptomatik im linken Bein bereits wieder komplett zurückgebildet.
Der Ehefrau des Patienten ist aufgefallen, dass ihr Mann in den letzten Wochen wesensverändert war und dass er zunehmende Gedächtnisstörungen entwickelt hat.

In der Notaufnahme wurde eine cCT durchgeführt ( $\triangleright$ Abb. 1). Es zeigte einen ausgedehnten Falx-ständigen Tumor rechts mit Umgebungsödem.
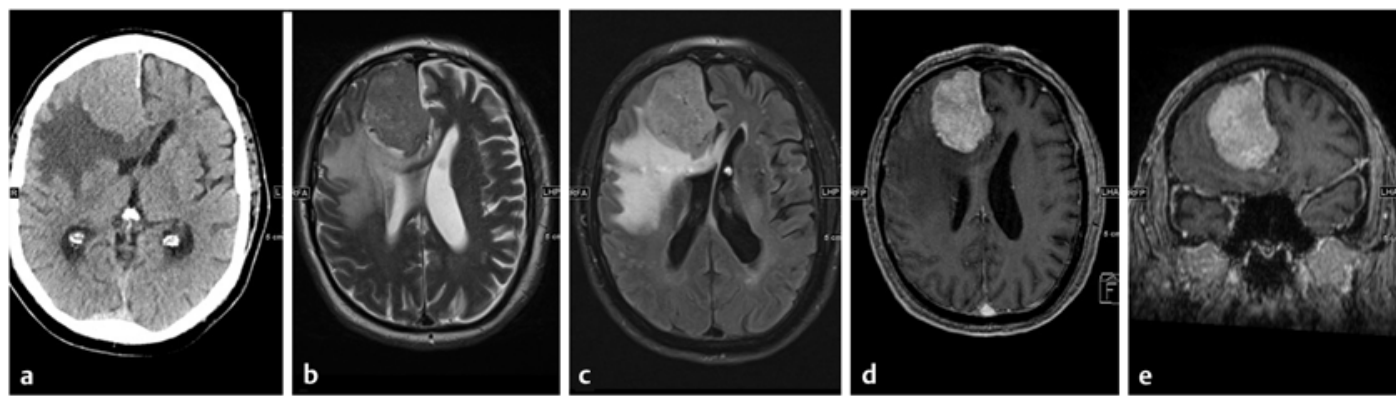

- Abb.1 Ausgedehntes rechtsseitiges Falxmeningeom mit angrenzendem Perifokalödem (CT- und MRT-Bilder mit freundlicher Genehmigung von Prof. Stefan Hähnel, Abt. Neuroradiologie, Universitätsklinikum Heidelberg). a Kraniale CT. b Kraniale MRT; T2-Wichtung. c Kraniale MRT; T2-FLAIR-Aufnahme. d Kraniale MRT; T1-KM-Aufnahme in axialer Schnittführung. e Kraniale MRT; T1-KM-Aufnahme in koronarer Schnittführung. 


$\begin{array}{ll}\text { ABKÜRZUNGEN } & \\ \text { AKT } & \text { Gen kodierend für die Proteinkinase B } \\ \text { ASXL1 } & \text { additional Sex Combs-like 1 } \\ \text { BAP1 } & \text { BRCA1-assoziiertes Protein (BRCA1 = Breast } \\ & \text { Cancer 1) } \\ \text { BRD4 } & \text { Bromodomän-haltiges Protein 4 } \\ \text { cCT } & \text { kraniale Computertomografie (Schädel-CT) } \\ \text { CMRT } & \text { kraniale Magnetresonanztomografie } \\ & \text { (Schädel-MRT) } \\ \text { DOTATOC } & \text { 1,4,7,10-Tetraazacyclododecan-1,4,7,10- } \\ & \text { Tetraessigsäure-Phe1-Tyr3-Octreotid (DOTA- } \\ & \text { Tyrosin-Konjugat mit Octreotid) } \\ \text { EEC } & \text { Elektroenzephalografie } \\ \text { ERK2 } & \text { Mitogen-aktivierte Proteinkinase 2 } \\ \text { FAK-Inhibitor } & \text { Inhibitor der fokalen Adhäsionskinase } \\ \text { FLAIR } & \text { Fluid attenuated Inversion Recovery } \\ \text { KM } & \text { Kontrastmittel } \\ \text { KMT2 D } & \text { Lysin-Methyltransferase 2D } \\ \text { Lu-177 } & \text { Lutetium-177 } \\ \text { MN1 } & \text { Meningiom 1 } \\ \text { mTOR } & \text { mechanistic Target of Rapamycin } \\ \text { NF-2 } & \text { Neurofibromin 2 } \\ \text { PD-L1 } & \text { Programmed Cell Death-Ligand 1 } \\ \text { PET-CT } & \text { Positronenemissionstomografie } \\ \text { PI3K } & \text { Phosphatidyl-Inositol-3-Kinase } \\ \text { PIK3CA } & \text { Phosphatidylinositol-4,5-Bisphosphate 3- } \\ \text { SMO-Mutation } & \text { smothened Mutation } \\ \text { TERT } & \text { Telomerase reverse Transkriptase } \\ \text { TRAF7 } & \text { World Health Organization } \\ \text { WHO } & \text { Y-90 }\end{array}$

\section{Epidemiologie}

Unter den gutartigen Hirntumoren sind Meningeome die häufigste Tumorentität. Mit einer Inzidenz von ca. 8 Fällen pro 100000 Personen pro Jahr machen diese etwa ein Drittel aller Hirntumoren aus [1]. Ihren Ursprung haben sie in den arachnoidalen Deckzellen. Meningeome treten häufiger bei Frauen als bei Männern auf, was insbesondere um das 40. Lebensjahr (Verhältnis 3:1) und bei spinalen Meningeomen deutlich wird, die zu $90 \%$ Frauen betreffen [2]. Das mittlere Erkrankungsalter liegt bei etwa 63 Jahren [3].

\begin{abstract}
Merke
Treten Meningeome in jüngerem Alter auf, muss an Risikofaktoren gedacht werden, beispielsweise eine vorausgegangene Strahlentherapie oder eine genetische Prädisposition.
\end{abstract}

Die Wahrscheinlichkeit eines strahleninduzierten Meningeoms nimmt mit der Zeit nach der Bestrahlung zu und erreicht über $8 \%$ nach 25 Jahren [4]. Zu den genetischen Erkrankungen, die für die Entstehung eines Meningeoms prädisponieren, gehört u.a. die Neurofibromatose Typ 2 (NF-2). Bei der NF-2 kommt es typischerweise durch Keimbahnmutationen des NF-2-Gens zur Entwicklung von bilateralen Akustikusneurinomen und Meningeomen [5].

\section{Einteilung von Meningeomen}

Gemäß der Lokalisation unterscheidet man

- Konvexitätsmeningeome,

- Falxmeningeome,

- Meningeome der Schädelbasis und

- spinale Meningeome.

Meningeome wachsen verdrängend gegenüber dem Gehirngewebe und arrodieren häufiger umgebende Knochenstrukturen oder auch das angrenzende Weichteilgewebe, was radiologisch als diagnostisches Kriterium genutzt wird. Bei einem Einwachsen in zerebrale Hirnvenen und Sinus kann es zu venösen Abflussstörungen und einer erheblichen Blutungsgefahr bei operativen Eingriffen kommen. Bei ca. 10\% der Patienten liegen multiple Meningeome vor [6].

\section{Merke}

Meningeome sind zumeist gutartig ( $80 \%$ ), können aber auch atypisch (15-20\%) oder anaplastisch $(1-3 \%)$ sein.

In die histologische Diagnose und Tumorgraduierung fließen verschiedene Faktoren ein [7]:

- der histologische Zelltyp ( Tab. 1),

- der mitotische Index,

- die Zellularität und

- das Vorhandensein von Nekrosen oder Hirnparenchyminfiltrationen. 
Zudem haben molekulare Veränderungen in den letzten Jahren zunehmend an Bedeutung bei der Einteilung von Meningeomen gewonnen, sind aber noch nicht Bestandteil der gültigen WHO-Klassifikation [8]. An spezialisierten Zentren wird dafür beispielsweise auf Hochdurchsatz-Methylierungsanalysen beispielsweise durch $850 k$-Arrays und Panel-Gensequenzierungen zurückgegriffen. Bei der 850k-Analyse wird das genomweite Methylierungsprofil des Tumormaterials eines Patienten mit dem von über 2800 Tumoren einer Referenzbank abgeglichen.

Ein „Classifier-Score“ drückt dabei die Ähnlichkeit mit einer Referenzgruppe aus und erlaubt in Zusammenschau mit klinischen Faktoren auch Rückschlüsse auf die Rezidivwahrscheinlichkeit des Meningeoms [9]. Klinisch nützlich sind diese Informationen vor allem bei den Meningeomen, die qua Histologie nicht eindeutig dem WHO Grad I zugeordnet werden können, oder allen, die dem WHO Grad II entsprechen, sodass sich die Frage einer adjuvanten Radiotherapie stellt. Während mit der DNA-Methylierungsanalyse die Tumorart und Veränderungen der Kopienzahl nachgewiesen werden können, ermöglicht die Panel-Sequenzierung den Nachweis der genauen Mutationen innerhalb der Gene und somit z. B. auch von Punktmutationen. Dabei wird eine Vorauswahl von zahlreichen mutmaßlich relevanten Genen parallel untersucht [10].

Eine Inaktivierung von Neurofibromin 2 (NF-2, Merlin) durch Monosomie 22 oder Mutationen tritt bei ca. $50 \%$ aller Meningeome auf [5]. Bei etwa 13\% der Patienten ohne NF-2 Veränderung sind Mutationen von AKT1 gefunden worden, was den Signalweg Phosphatidyl-Inositol-3-Kinase (PI3K)/AKT/mechanistic Target of Rapamycin (mTOR) beeinflusst. Bei weiteren $5 \%$ der Patienten ohne NF-2-Alteration wurden smothened (SMO)-Mutationen identifiziert, die Teil des Hedgehog-Signalweges sind. Interessanterweise sind über $60 \%$ der Meningeome mit AKT1 oder SMO-Mutationen an der Schädelbasis lokalisiert ( $\triangleright$ Abb. 2) [12].
- Tab. 1 Histopathologische Einteilung von Meningeomen und assoziierte Genmutationen gem. Preusser et al. [11].

\begin{tabular}{|c|c|c|}
\hline Diagnose & $\begin{array}{l}\text { WHO } \\
\text { Grad }\end{array}$ & Genmutationen \\
\hline \multicolumn{3}{|l|}{ Benigne Meningeome } \\
\hline meningoepitheliale Meningeome & 1 & $\begin{array}{l}\text { TRAF7, AKT1, POLR2A, } \\
\text { PIK3CA }\end{array}$ \\
\hline fibroblastische Meningeome & 1 & $N F-2$ \\
\hline transitionale Meningeome & 1 & NF-2, AKT1, PIK3CA \\
\hline psammomatöse Meningeome & I & $N F-2$ \\
\hline angiomatöse Meningeome & 1 & - \\
\hline mikrozystische Meningeome & I & - \\
\hline sekretorische Meningeome & 1 & KLF4, TRAF7 \\
\hline lymphoplasmazellreiche Meningeome & 1 & - \\
\hline metaplastische Meningeome & 1 & - \\
\hline \multicolumn{3}{|l|}{ Nicht-benigne Meningeome } \\
\hline chordoidales Meningeom & II & - \\
\hline klarzellige Meningeome & II & SMARCE1 \\
\hline atypische Meningeome & II & NF-2, TRAF7, AKT1 \\
\hline papilläre Meningeome & III & - \\
\hline rhabdoide Meningeome & III & BAP1 \\
\hline anaplastische (maligne) Meningeome & III & $N F-2$ \\
\hline
\end{tabular}

Bestimmten Subtypen von Meningeomen konnten weitere Mutationen zugeordnet werden [11]. So wurden bei sekretorischen Meningeomen kombinierte Mutationen von Kruppel-like factor 4 (KLF4) K4090Q und Tumornekrosefaktor-assoziierter Faktor 7 (TRAF7) gefunden, die nie gleichzeitig mit NF-2-Mutationen auftraten. Bei rhabdoiden Meningeomen ist eine Inaktivierung des Tumorsuppressorgens BRCA1-assoziiertes Protein (BAP)1 mit einer signifikant kürzeren rezidivfreien Zeit assoziiert. Ferner treten PIK3CA und Telomerase reverse Transkriptase (TERT)-Promoter Mutationen häufiger bei Meningeomen auf. Letztere sind dort mit einer schlechteren Prognose assoziiert [13]. 


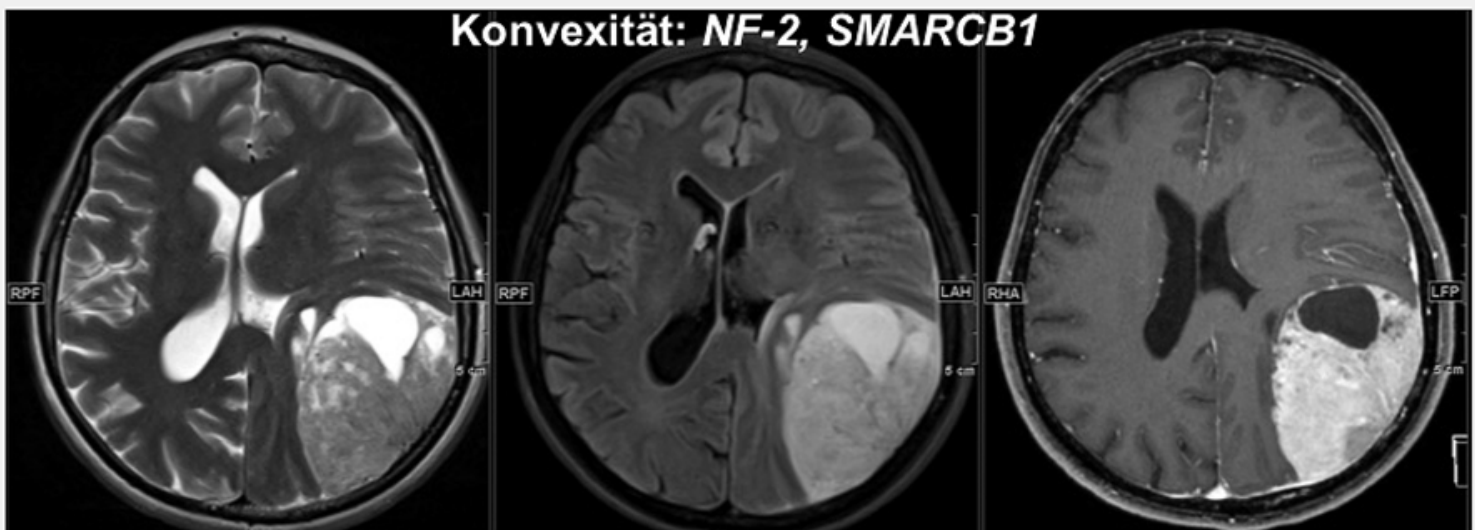

Schädelbasis: AKT-1, SMO, TRAF7, KLF4, POLR2A, PIK3CA
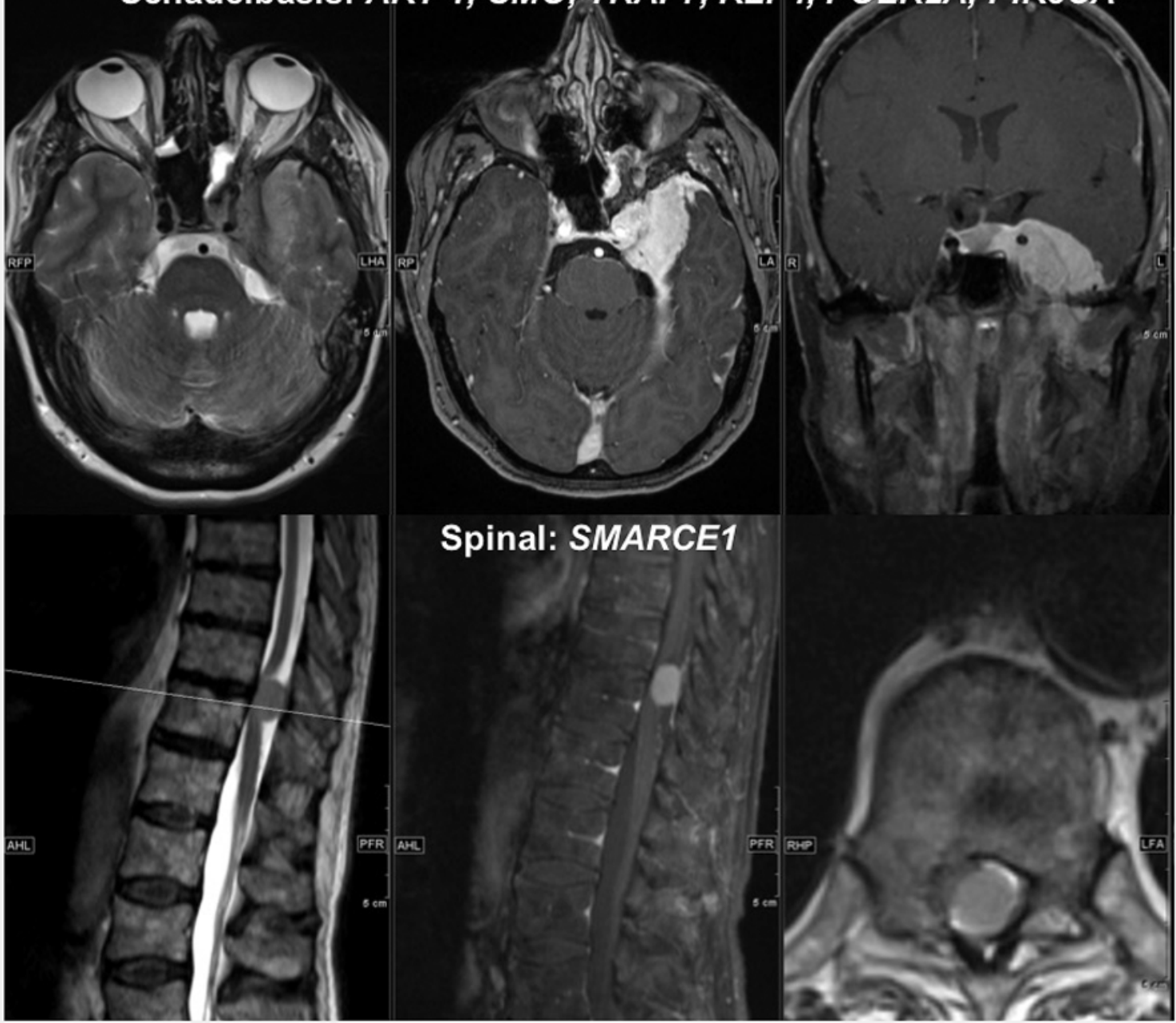

Abb.2 Genetische Alterationen bei Meningeomen und deren lokalisatorische Zuordnung. Magnetresonanztomografie (MRT) mit T2- (jeweils links), T2-FLAIR (Fluid-Attenuated-Inversion-Recovery, oben, Mitte) und T1-Aufnahmen nach Kontrastmittelgabe (KM) zeigen exemplarische Meningeome an unterschiedlichen Tumorlokalisationen. Zu bemerken ist die angedeutete Radspeichenstruktur beim dargestellten Konvexitätsmeningeom. Typische Genveränderungen, die mit den jeweiligen Tumorlokalisationen assoziiert sind, sind jeweils kursiv angegeben (MRT-Bilder mit freundlicher Genehmigung von Prof. Stefan Hähnel, Abt. Neuroradiologie, Universitätsklinikum Heidelberg). 


\section{Symptome und Diagnostik}

Merke

Meningeome bleiben aufgrund ihres langsamen Wachstums häufig lange Zeit symptomlos. In der Folge werden die meisten Meningeome auch als $\mathrm{Zu}$ fallsbefund detektiert.

Oft werden sie zufällig erkannt, wenn eine zerebrale Bildgebung erfolgt, z.B. im Rahmen einer Kopfschmerzabklärung. Je nach Größe und Lokalisation können allgemeine Symptome von Hirntumoren auftreten, wie z.B. epileptische Anfälle oder Hirndrucksymptome. Bei einer Lokalisation an der Falx cerebri kann ein Mantelkantensyndrom imponieren. Dabei kommt es zu einer Paraparese der Beine und zu unkontrollierter Blasenentleerung. Olfaktoriusrinnen-Meningeome führen in der Regel zu einer Hyposmie oder Parosmie. Bei Keilbeinflügelmeningeomen kann es durch Druck auf den N. opticus zu einer Visusstörung kommen. Treten dabei eine ipsilaterale Optikusatrophie und eine kontralaterale Stauungspapille auf, spricht man auch vom sog. Foster-Kennedy-Syndrom. Bei Meningeomen am Kleinhirnbrückenwinkel sind Ausfälle des V., VII. und VIII. Hirnnervs möglich. Demgegenüber können spinale Meningeome zu Paresen und Sensibilitätsstörungen bis hin zum Querschnittssyndrom führen.

Während der Schwangerschaft kann es zu einem deutlichen Wachstum von Meningeomen kommen. Neben plausiblen hormonellen Einflüssen könnte dabei auch eine Gewebelockerung mit intra- und interzellulärem Ödem eine Rolle spielen [14].

In der Computertomografie des Schädels stellen sich Meningeome nativ scharf abgegrenzt, hyperdens, selten isodens dar. Häufig zeigen sich Verkalkungen. Nach Gabe von Kontrastmittel ist meist eine intensive homogene Kontrastmittelaufnahme nachweisbar (Bild eines „Schneeballs“).

\section{Merke}

Goldstandard in der Diagnostik von Meningeomen ist die MRT.

Am eindeutigsten kann eine Infiltration des Knochens aber mittels einer dünnschichtigen $\mathrm{CT}$ identifiziert werden. In der MRT sind Meningeome in der T1-Wichtung in der Regel hypointens, während sie in der T2Wichtung iso- bis hyperintens sind.
PRAXIS

Tipp

Ein klassischer Befund für ein Meningeom ist das „Dural Tail Sign“ [15], welches für einen breiten Durakontakt des Meningeoms mit Verdickung der Randzone steht, sodass es aussieht, als hätte das Meningeom einen Ausläufer.

Ferner kann es durch größere Tumorgefäße zu punktförmigen Signalauslöschungen kommen, die als Flow Voids bezeichnet werden. Ein Meningeom der mittleren Schädelgrube mit starker ossärer Komponente wird auch als Meningeom en plaque bezeichnet.

Mithilfe der MR-Angiografie lässt sich die Lagebeziehung zu großen Venen oder Sinus darstellen oder auch eine Ummauerung von intrazerebralen Arterien. Die konventionelle Angiografie hat einen (seltenen) Stellenwert in der Diagnostik von Meningeomen, wenn eine präoperative Embolisation oder ein geplanter Verschluss bzw. eine Rekonstruktion größerer Arterien geplant ist.

Bei diagnostisch unklaren Fällen kann eine Octreotidrezeptorszintigrafie hilfreich sein [16]. Dabei werden Meningeome anhand ihrer Somatostatin-Rezeptoren dargestellt, um sie gegenüber anderen Tumoren oder entzündlichen Läsionen, wie z.B. Metastasen oder einer Sarkoidose, abzugrenzen.

\section{FALLBEISPIEL \\ Fall 1 (Fortsetzung)}

Der Patient wurde zur weiteren Diagnostik inklusive CMRT und EEG stationär aufgenommen. In der ergänzenden cMRT erhärtete sich der Verdacht auf ein Falxmeningeom, und der Patient wurde in der neurochirurgischen Klinik zur Resektion vorgestellt.

Es erfolgte eine mikrochirurgische Exzision des rechtsfrontalen Meningeoms mit regelrechtem postoperativem Verlauf. Histologisch zeigte sich ein Meniningeom WHO-Grad I vom transitionalen Subtyp.

Der Patient konnte in gutem Allgemeinzustand in die ambulante Behandlung entlassen werden, mit der Empfehlung, sich nach 3 Monaten mit aktuellen cMRT-Bildern in der neurochirurgischen Ambulanz vorzustellen. 


\section{Aktuelle Therapieoptionen}

Trotz der relativen Häufigkeit von Meningeomen liegen aktuell - wie bei anderen Hirntumoren leider auch keinerlei Ergebnisse von prospektiven randomisierten klinischen Studien zum Stellenwert der Operation und im Falle von Meningeomen auch nicht der Strahlentherapie vor.

Merke

Grundsätzlich sollte die Behandlung von Patienten mit symptomatischen Meningeomen im Rahmen einer interdisziplinären Tumorkonferenz besprochen werden.

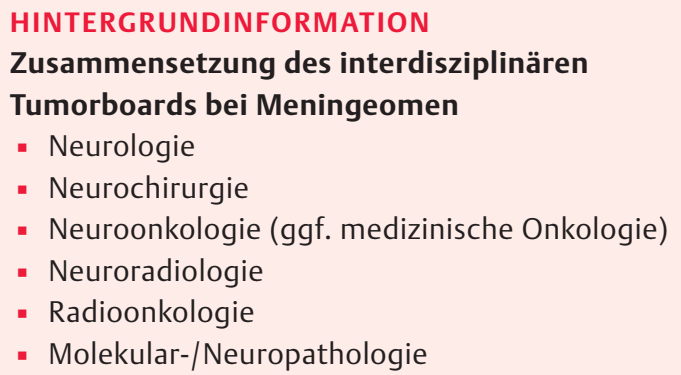

\section{Chirurgische Therapie}

Bei kleinen Tumoren in funktionell unproblematischer Lage kann insbesondere bei älteren Patienten ein abwartendes Verhalten mit regelmäßigen Verlaufskontrollen indiziert sein [17].

Merke Ansonsten ist die Standardtherapie von Meningeomen die Tumorresektion. Das Ziel der Operation ist eine möglichst komplette Entfernung des Tumors sowie der befallenen Hirnhaut und Knochenstrukturen [18].

Neben der histopathologischen Sicherung zielt die Resektion auf eine Symptomlinderung ab. Rezidive können insbesondere bei schädelbasisnahen Tumoren und inkompletter Resektion vorkommen. In diesen Fällen werden daher engmaschige MRT-Kontrollen gegenüber einer postoperativen Strahlentherapie abgewogen.
Der operative Zugang hängt von der Tumorlokalisation ab. Grundsätzlich wird ein Zugangsweg gewählt, der eine möglichst optimale Darstellung des Tumors, der involvierten Meningen und angrenzenden neurovaskulären Strukturen bietet. Bei tiefliegenden Meningeomen bieten sich verschiedene transossäre Zugänge an der Schädelbasis an.

Bei gefäßreichen Tumoren kann eine präoperative Embolisation sinnvoll sein. Eine frühe Identifizierung und der Verschluss der tumorversorgenden Gefäße ist für die Übersichtlichkeit im Operationsfeld entscheidend. Anschließend erfolgt ein „Aushöhlen“ des Tumors und im Verlauf eine sorgfältige Präparation und Entfernung der Tumorkapsel unter Schonung neurovaskulärer Strukturen. Für ein gutes postoperatives Ergebnis sind ein intraoperatives Neuromonitoring, stereotaktische Navigationssysteme und eine endoskopische Unterstützung sehr hilfreich [19,20].

Bei Beteiligung der Sinus muss sorgfältig zwischen einem möglichst radikalen Operationsergebnis und möglichen Komplikationen durch venöse Infarkte abgewogen werden.

\section{PRAXIS}

Tipp

Unter Umständen kann es sinnvoll sein, einen infiltrierten Sinus in situ zu belassen, wobei sich durch den langsamen Verschluss venöse Kollateralkreisläufe bilden und der Sinus anschließend bei Bedarf en bloc entfernt werden kann.

In der postoperativen Phase muss besonderes Augenmerk auf eine ausreichende Thromboseprophylaxe gerichtet werden, da in bis zu 32\% der Fälle tiefe Beinvenenthrombosen und Lungenembolien auftreten [21].

\section{Strahlentherapie}

Bei ungünstiger Lage des Meningeoms sollten strahlentherapeutische Behandlungsoptionen in Betracht gezogen werden. Vergleichende Untersuchungen unterschiedlicher strahlentherapeutischer Verfahren und Techniken gibt es nicht. Dies gilt für Gamma oder Cyber Knife und auch die Partikelstrahlung (s. a. Infobox). 
PRAXIS

\section{Radiochirurgische Therapie}

Radiochirurgische Verfahren (Gamma-Knife) sind insbesondere bei kleinen, symptomatischen Meningeomen (z. B. Durchmesser $<3 \mathrm{~cm}$, Volumen $<10 \mathrm{~cm}^{3}$ ) als primäre Behandlung etabliert [22]. Ansonsten werden sie auch postoperativ bei inkomplett entfernten Tumoren oder in der Rezidivsituation angewendet. Bei einer Einzeldosis von 13-15 Gy kann eine lokale Tumorkontrolle von über $90 \%$ der Patienten erreicht werden [23]. Nachteil der primär radiochirurgischen Therapie ist die fehlende histologische Sicherung des Tumors.

\section{Merke}

Eine stereotaktische fraktionierte Radiotherapie ist unabhängig von der Tumorgröße primär oder postoperativ einsetzbar und bei malignen Meningeomen postoperativ immer indiziert.

Bei unmittelbarer Nähe zum optischen System ist die stereotaktische fraktionierte Radiotherapie der Radiochirurgie vorzuziehen. Das Bestrahlungszielvolumen beinhaltet den Tumor mit minimalem Sicherheitssaum in einer Gesamtdosis von 45-60 Gy in Einzeldosen von 1,8-2Gy, entsprechend dem Malignitätsgrad des Tumors. Auch mit der stereotaktischen fraktionierten Radiotherapie kann eine sehr gute lokale Tumorkontrolle $(>90 \%)$ bei WHO-Grad-I- und -II-Meningeomen erreicht werden [24]. Inwieweit eine adjuvante Strahlentherapie bei komplett entfernten WHO-Grad-II-Meningeomen sinnvoll ist, wird aktuell in einer internationalen Studie untersucht [25].

An entsprechend ausgestatteten Zentren wird vermehrt auf eine Partikelstrahlentherapie mit Protonen oder Kohlenstoffionen zur Behandlung rezidivierter, atypischer oder anaplastischer Meningeome zurückgegriffen. Bei der Partikeltherapie soll das dem Tumor umliegende Gehirngewebe besser als bei einer konventionellen Photonenbestrahlung geschont werden, was insbesondere auch bei chirurgisch schlecht zugänglichen Meningeomen der Schädelbasis von Interesse ist [26].
In einer aktuellen Metaanalyse von 12 Studien zur Partikeltherapie bei Meningeomen konnte eine vergleichbare lokale Tumorkontrolle bei Protonen-, Kohlenstoffionen und Photonenbestrahlung beobachtet werden [27]. Inwieweit eine bessere klinische Verträglichkeit mit z.B. geringeren neurokognitiven Defiziten durch die Partikeltherapie erreicht werden kann, sollte in randomisierten klinischen Studien untersucht werden.

\section{Medikamentöse Therapie}

\section{Merke}

Die medikamentöse Tumortherapie kommt üblicherweise erst in Frage, wenn lokale Therapieverfahren versagen.

In kleinen retrospektiven Fallserien zeigte der Angiogenese-Hemmstoff Bevacizumab eine moderate Wirksamkeit bei rezidivierten oder progredienten Meningeomen [28]. Für den oralen MultikinaserezeptorHemmstoff Sunitinib bestehen ebenfalls Hinweise auf Wirksamkeit bei therapierefraktären WHO Grad-IIund -III-Meningeomen, bei teils aber lebensbedrohlichen Nebenwirkungen wie schweren Hirnblutungen [29] und ohne ausreichende Stringenz der Serien und bei Fehlen einer Kontrollintervention.

\section{Sonstige}

Im Rahmen von individuellen Heilversuchen kann bei Patienten mit Somatostatin-Rezeptor-positiven, progredienten Meningeomen eine zielgerichtete Radionuklidtherapie in Erwägung gezogen werden. In einer retrospektiven Evaluation von 20 Patienten mit einer Lu-177- und Y-90-DOTATOC-Therapie konnte bei 10 von 20 Patienten eine Stabilisierung der Erkrankung erreicht werden. Innerhalb der Serie wurde ein längeres progressionsfreies Überleben bei Patienten mit einer hohen Expression von Somatostatin-Rezeptoren beobachtet [30]. 


\section{FALLBEISPIEL}

Fall 2

Eine 34-jährige Patientin stellte sich aufgrund rezidivierender Kopfschmerzen im Mai 2016 in der neurologischen Notambulanz vor. Bei der Patientin waren aufgrund einer akuten lymphatischen Leukämie im Kindesalter eine Chemotherapie und Ganzhirnbestrahlung durchgeführt worden. Klinisch-neurologisch war die Patientin zum Vorstellungszeitpunkt unauffällig.

Es erfolgte eine cMRT zum Ausschluss einer symptomatischen Kopfschmerzursache, welche schließlich eine hochgradig meningeomverdächtige Raum- forderung, flächig im Bereich der Konvexität, von postzentral bis zum Tentorium reichend zeigte

( $>$ Abb. 3).

Es erfolgte eine inkomplette mikrochirurgische Tumorresektion nach osteoplastischer Trepanation von bipostzentral bis okzipital reichend. Die histopathologische Aufarbeitung ergab ein Meningeom, WHO Grad I.

Die Patientin erholte sich postoperativ gut und konnte ohne fokal-neurologische Defizite wieder in die Häuslichkeit entlassen werden.
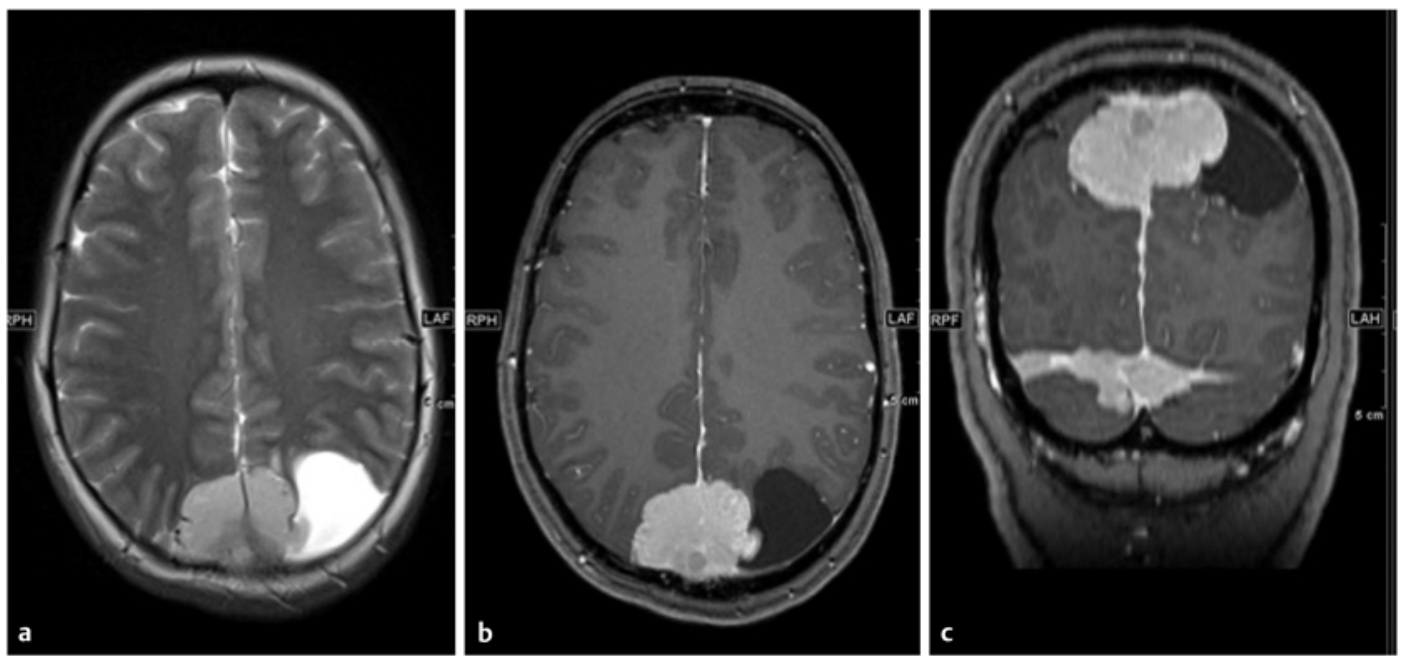

- Abb. 3 Hochgradig meningeomverdächtige Raumforderung, flächig im Bereich der Konvexität, von postzentral bis zum Tentorium reichend (MRT-Bilder mit freundlicher Genehmigung von Prof. Stefan Hähnel, Abt. Neuroradiologie, Universitätsklinikum Heidelberg). a MRT mit T2-Aufnahme. b MRT mit T1-KM-Aufnahmen in axialer Schnittführung. c MRT mit T1-KM-Aufnahmen in koronarer Schnittführung.

\section{Therapieentwicklung}

\section{Trabectedin}

Basierend auf vielversprechenden experimentellen Daten wurde Trabectedin in einer randomisierten, multizentrischen Phase-III-Studie bei rezidivierten WHOGrad-II- und -III-Meningeomen untersucht (EORTC1320-BTG). Trabectedin ist ein ursprünglich aus gallertartigen Meerestieren entwickelter Wirkstoff, der über einen komplexen Wirkmechanismus die DNA-AIkylierung und Reparaturmechanismen sowie das Mikromilieu der Tumorzellen beeinflusst. Erste Auswertungen der Studie deuten allerdings nicht auf eine Wirksamkeit von Trabectedin hin. Die Therapie mit Trabectedin wurde zu einer Behandlung nach lokalem Therapiestandard verglichen. Interessanterweise waren dabei im nicht experimentellen Arm möglicher- weise erneut Effekte einer antiangiogenen Therapie zu sehen [31].

\section{Zielgerichtete Therapieansätze}

Basierend auf den zunehmenden Fortschritten in der molekularen Charakterisierung von Meningeomen werden aktuell vermehrt auch zielgerichtete Therapieansätze bei Meningeomen untersucht [32]. So werden in einer aktuellen Phase-II-Studie SMO, AKT1 und FAKInhibitoren hinsichtlich ihrer Wirksamkeit bei rezidivierten oder progredienten Meningeomen mit den entsprechenden SMO-, AKT1 - oder NF-2-Mutationen untersucht (NCT02523014) 


\section{FALLBEISPIEL}

\section{Fall 2 (Fortsetzung)}

Bei erheblichem Tumorprogress mit deutlich raumfordernder Wirkung innerhalb der hinteren Schädelgrube erfolgte im März 2017 eine weitere Resektion der infratentoriellen Tumorrestanteile in mikrochirurgischer Technik. Weiterhin konnte keine Komplettresektion erreicht werden. Aufgrund der Ausdehnung und der kritischen Nachbarorgane wurde daher eine ergänzende Präzisionsstrahlentherapie geplant. Dabei erfolgte eine definitive Partikeltherapie der Schädelbasis und der Falx okzipital mit Protonen in einer Gesamtdosis von 54 Gy in 27 Fraktionen.

Im Mai 2019 waren schließlich progrediente Meningeomherde mit kompletter Durchsetzung des Sinus sagittalis superior und beider Sinus transversus nachweisbar. Bei fehlenden chirurgischen und strahlentherapeutischen Therapieoptionen wurde ein Therapieversuch mit Bevacizumab im freien Heilversuch beantragt und auch von der Krankenkasse zeitlich befristet genehmigt. Zugleich erfolgte eine Panel-Sequenzierung und 850k-Analyse zur Aufarbeitung des Tumormaterials. Im vorliegenden Patientenfall ergab der Meningeom-Classifier die Methylierungsklasse Meningeom, mit höchstem Classifier-Score für die Subklasse intermediär A [38], Mitogen-aktivierte Proteinkinase (ERK)2-amplifiziert und Bromodomän-haltiges Protein (BRD)4-amplifiziert. Über die Histologie eines Meningeoms WHO Grad I hinaus konnten somit molekulare Hinweise auf eine erhöhte Rezidivneigung erhoben werden.
Mithilfe der Panel-Sequenzierung ließ sich noch eine additional Sex Combs-like (ASXL)1, Lysin-Methyltransferase (KMT)2D und Meningiom (MN)1-Mutation unklarer Relevanz detektieren.

Im Dezember 2019 war schließlich eine erneute Befundprogredienz unter der antiangiogenen Therapie mit Bevacizumab nachweisbar. Es erfolgte eine Vorstellung im interdisziplinären Tumorboard. Wegen der bereits erreichten maximalen sinnvollen Dosis konnte keine weitere Strahlentherapie empfohlen werden, ebenso wäre eine erneute Resektion der aktuell asymptomatischen Patientin möglicherweise mit einer hohen Morbidität verbunden gewesen. Die $850 \mathrm{k}$-Analyse und Panel-Sequenzierung hatte keine Alteration ergeben, die mit einer zielgerichteten Therapie angreifbar wäre. Zur Evaluation eines individuellen Heilversuches mit DOTATOC wurde bei der Patientin ein DOTATOC-PET-CT mit der Frage nach Expression von Somatostatinrezeptoren durchgeführt.

Nachdem das DOTATOC-PET eine kräftige Nuklidspeicherung im Tumorareal angezeigt hatte ( $\triangleright$ Abb.4), erfolgte im Rahmen eines individuellen Heilversuches eine DOTATOC-Therapie mit 2 GBq Lu-177 und 2,5 GBq Y-90 über zunächst 2 Zyklen. Die Patientin war unter dieser Therapie zuletzt klinisch und bildgebend in der kurzfristigen Kontrolle stabil.
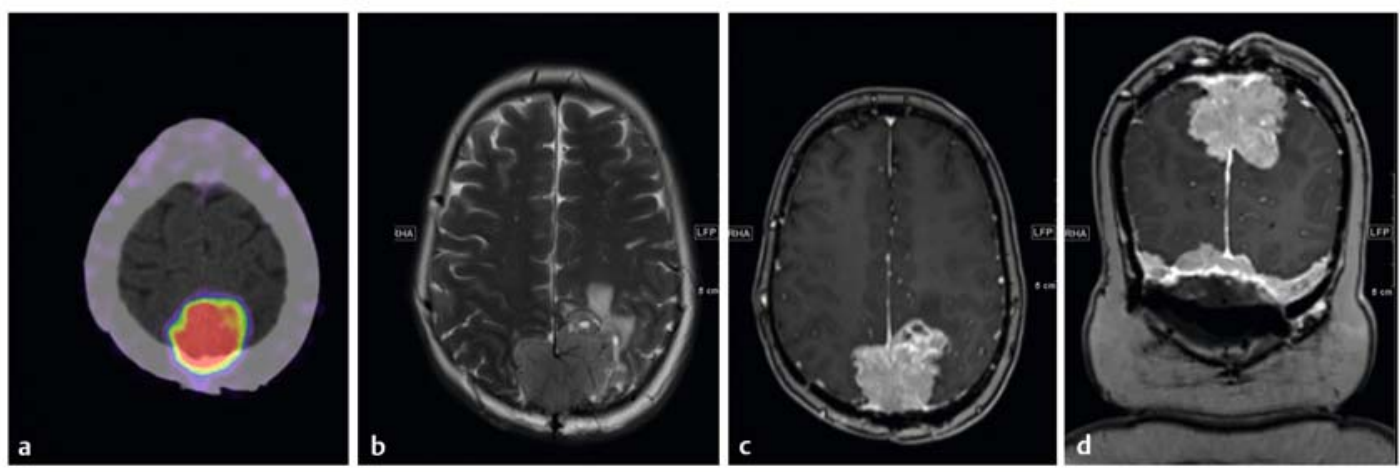

- Abb. 4 Kräftige Nuklidspeicherung im Tumorareal. (PET-CT-Bilder mit freundlicher Genehmigung von Prof. Stefan Hähnel, Abt. Neuroradiologie, Universitätsklinikum Heidelberg) a DOTATOC-PET-CT. b Kraniale MRT; T2-FLAIR-Aufnahme. c Kraniale MRT; T1-KM-Aufnahmen in axialer Schnittführung. d Kraniale MRT; T1-KM-Aufnahmen in koronarer Schnittführung. 


\section{Immuntherapie}

Die Rolle von Immuntherapien zur Behandlung von Meningeomen ist aktuell - wie auch sonst in der Neuroonkologie - noch nicht ausreichend geklärt. Bei anaplastischen Meningeomen wurde eine erhöhte Expression des Programmed Cell Death-Ligand 1 (PD-L1) beobachtet, was zu einer Verminderung von infiltrierenden T-Lymphozyten und einem immunsuppressiven Tumormikromilieu führte. Pembrolizumab wird aktuell bei rezidivierten oder residuellen hochgradigen Meningeomen in einer Phase-II-Studie untersucht (NCT03279692).

\section{Nachsorge und Prognose}

\section{Merke}

Die postoperative Nachsorge besteht üblicherweise aus einer zunächst einmaligen Kontrolle nach 3 Monaten gefolgt von jährlichen Verlaufskontrollen mittels klinischer Untersuchung und MRT über zumindest 10 Jahre.

Die Rezidivrate von Patienten mit Meningeomen hängt stark ab

- vom WHO-Grad,

- dem Ausmaß der chirurgischen Resektion und

- von einer etwaigen postoperativen Strahlentherapie.

1957 beschrieb Donald Simpson erstmals Rezidivraten von Meningeomen entsprechend dem Resektionsausmaß [33]. Auch in aktuelleren Studien wurden bei komplett entfernten WHO-Grad-I-Meningeomen Rezidivraten von ca. $20 \%$ nach 10 Jahren beschrieben, wohingegen bei inkomplett entfernten Tumoren Rezidivraten von über $50 \%$ im gleichen Zeitraum beobachtet wurden $[34,35]$. Mithilfe der postoperativen Strahlentherapie kann die 10-Jahres-Rezidivrate von unvollständig entfernten Meningeomen auf 33\% reduziert werden [36].

\section{Merke}

Die 10-Jahres-Überlebensrate von Patienten mit malignen Meningeomen beträgt nur ca. $35 \%$ [37], weshalb eine weitere Erforschung alternativer Behandlungsmöglichkeiten dringend notwendig ist.

\section{KERNAUSSAGEN}

- Bildgebung der Wahl bei Verdacht auf ein Meningeom ist die kontrastmittelgestützte MRT. Das cCT kann zur Beurteilung der Knocheninfiltration wertvoll sein.

- Kleine asymptomatische Meningeome bei älteren und vorerkrankten Patienten können verlaufskontrolliert werden, ansonsten ist prinzipiell eine histologische Sicherung anzustreben.

- Die Behandlung von symptomatischen Meningeomen sollte in einem interdisziplinären Tumorboard festgelegt werden.

- Goldstandard ist die möglichst komplette operative Tumorresektion.

- Bei inkomplett entfernten atypischen Meningeomen sollte eine adjuvante Bestrahlung erfolgen.

- Bei anaplastischen Meningeomen sollte immer eine adjuvante Bestrahlung erfolgen.

- Die medikamentöse Tumortherapie hat bisher keinen etablierten Stellenwert zur Behandlung von Meningeomen.

- Kleinere Studien ergaben Hinweise auf eine Wirksamkeit von antiangiogenen Substanzen zur Behandlung von therapierefraktären Meningeomen.

- In aktuellen Studien werden molekular zielgerichtete Substanzen u. a. gegen NF-2, AKT1 und SMO zur Behandlung von Meningeomen untersucht.

\section{Schlüsselwörter}

Hirntumor, Meningeom, NF-2, zielgerichtete Therapie

Interessenkonflikt

Erklärung zu finanziellen Interessen

Forschungsförderung erhalten: nein; Honorar/geldwerten Vorteil für Referententätigkeit erhalten: nein; Bezahlter Berater/interner Schulungsreferent/Gehaltsempfänger: nein; Patent/Geschäftsanteile/Aktien (Autor/Partner, Ehepartner, Kinder) an Firma (Nicht-Sponsor der Veranstaltung): nein; Patent/Geschäftsanteile/Aktien (Autor/Partner, Ehepartner, Kinder) an Firma (Sponsor der Veranstaltung): nein.

Erklärung zu nichtfinanziellen Interessen

Die Autorinnen/Autoren geben an, dass kein Interessenkonflikt besteht. 


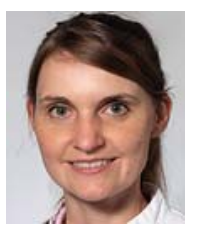

\section{Corinna Seliger}

PD Dr. med., Jahrgang 1985. 2005-2011 Studium der Humanmedizin an der Universität Regensburg. 2012-2019 Facharztausbildung Neurologie. Seit 10/2019 Oberärztin in der Neurologie am Universitätsklinikum Heidelberg. Schwerpunkt: Neuroonkologie.

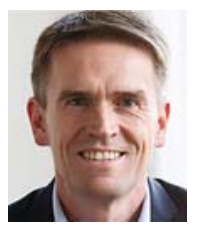

\section{Wolfgang Wick}

Prof. Dr. med., Ärztlicher Direktor der Neurologischen Universitätsklinik in Heidelberg und Leiter der Klinischen Kooperationseinheit Neuroonkologie am Deutschen Krebsforschungszentrum. Medizinstudium 19901997 in Bonn, London und Boston. Facharztanerkennung und Habilitation 2003 nach Ausbildung in der Neurologie in Tübingen. Nach Tätigkeit als Oberarzt und Stellv. Ärztlicher Direktor in Tübingen von 2007-2014 Professor für Neuroonkologie und Ärztlicher Direktor der Abteilung Neuroonkologie in Heidelberg.

\section{Korrespondenzadresse}

\author{
Prof. Dr. med. Wolfgang Wick \\ Neurologische Klinik \\ Universitätsklinikum Heidelberg \\ Im Neuenheimer Feld 400 \\ 69120 Heidelberg \\ Deutschland \\ wolfgang.wick@med.uni-heidelberg.de
}

Wissenschaftlich verantwortlich gemäß

Zertifizierungsbestimmungen

Wissenschaftlich verantwortlich gemäß Zertifizierungsbestimmungen für diesen Beitrag ist Prof. Dr. med. Wolfgang Wick, Heidelberg.

\section{Literatur}

[1] Ostrom QT, Cioffi G, Gittleman H et al. CBTRUS Statistical report: primary brain and other central nervous system tumors diagnosed in the United States in 2012-2016. NeuroOncology 2019; 21: v1-v100. doi:10.1093/neuonc/noz150

[2] Cordera S, Bottacchi E, D’Alessandro G et al. Epidemiology of primary intracranial tumours in NW Italy, a population based study: stable incidence in the last two decades. J Neurol 2002; 249: 281-284. doi:10.1007/s004150200005

[3] Holleczek B, Zampella D, Urbschat S et al. Incidence, mortality and outcome of meningiomas: A population-based study from Germany. Cancer Epidemiol 2019; 62: 101562. doi:10.1016/j.canep.2019.07.001

[4] Strojan P, Popovic M, Jereb B. Secondary intracranial meningiomas after high-dose cranial irradiation: report of five cases and review of the literature. Int J Radiat Oncol Biol Phys 2000; 48: 65-73. doi:10.1016/s0360-3016(00) 00609-x
[5] Lekanne DeprezRH, Bianchi AB, Groen NA et al. Frequent NF2 gene transcript mutations in sporadic meningiomas and vestibular schwannomas. Am J Hum Genet 1994; 54 : 1022-1029

[6] Huang H, Buhl R, Hugo HH et al. Clinical and histological features of multiple meningiomas compared with solitary meningiomas. Neurol Res 2005; 27: 324-332. doi:10.1179/ $016164105 \times 39932$

[7] Louis DN, Perry A, Reifenberger G et al. The 2016 World Health Organization classification of tumors of the central nervous system: a summary. Acta Neuropathol 2016; 131: 803-820. doi:10.1007/s00401-016-1545-1

[8] Lee YS, Lee YS. Molecular characteristics of meningiomas. J Pathol Transl Med 2020; 54: 45-63. doi:10.4132| jptm.2019.11.05

[9] Nassiri F, Mamatjan $Y$, Suppiah S et al. DNA methylation profiling to predict recurrence risk in meningioma: development and validation of a nomogram to optimize clinical management. Neuro-Oncology 2019; 21: 901-910. doi:10.1093/neuonc/noz061

[10] Sahm F, Schrimpf D, Jones DT et al. Next-generation sequencing in routine brain tumor diagnostics enables an integrated diagnosis and identifies actionable targets. Acta Neuropathol 2016; 131: 903-910. doi:10.1007/s00401015-1519-8

[11] Preusser M, Brastianos PK, Mawrin C. Advances in meningioma genetics: novel therapeutic opportunities. Nat Rev Neurol 2018; 14: 106-115. doi:10.1038/nrneurol.2017.168

[12] Brastianos PK, Horowitz PM, Santagata S et al. Genomic sequencing of meningiomas identifies oncogenic SMO and AKT1 mutations. Nat Genet 2013; 45: 285289doi:10.1038/ng.2526

[13] Sahm F, Schrimpf D, Olar A et al. TERT Promoter Mutations and Risk of Recurrence in Meningioma. J Natl Cancer Inst 2016: 108. doi:10.1093/jnci/djv377

[14] Lusis EA, Scheithauer BW, Yachnis AT et al. Meningiomas in pregnancy: a clinicopathologic study of 17 cases. Neurosurgery 2012; 71: 951-961. doi:10.1227/NEU.0b013e $31826 a d f 65$

[15] Qi ST, Liu Y, Pan J et al. A radiopathological classification of dural tail sign of meningiomas. J Neurosurg 2012; 117: 645653. doi:10.3171/2012.6.JNS111987

[16] Afshar-Oromieh A, Giesel FL, Linhart HG et al. Detection of cranial meningiomas: comparison of (6)(8)Ga-DOTATOC PET/CT and contrast-enhanced MRI. Eur J Nucl Med Mol Imaging 2012; 39: 1409-1415. doi:10.1007/s00259-0122155-3

[17] Sughrue ME, Rutkowski M], Aranda D et al. Treatment decision making based on the published natural history and growth rate of small meningiomas. J Neurosurg 2010; 113: 1036-1042. doi:10.3171/2010.3.JNS091966

[18] Al-Mefty O, Holoubi A, Rifai A et al. Microsurgical removal of suprasellar meningiomas. Neurosurgery 1985; 16: 364372. doi:10.1227/00006123-198503000-00014

[19] Omay SB, Barnett GH. Surgical navigation for meningioma surgery. J Neuro-Oncology 2010; 99: 357-364. doi:10.1007/s11060-010-0359-6

[20] Topsakal C, Al-Mefty O, Bulsara KR et al. Intraoperative monitoring of lower cranial nerves in skull base surgery: technical report and review of 123 monitored cases. Neurosurg Rev 2008; 31: 45-53. doi:10.1007/s10143-007-0105-5 
[21] Hoefnagel D, Kwee LE, van Putten EH et al. The incidence of postoperative thromboembolic complications following surgical resection of intracranial meningioma. A retrospective study of a large single center patient cohort. . Clin Neurol Neurosurg 2014; 123: 150-154. doi:10.1016/j.clineuro.2014.06.001

[22] Pollock BE, Stafford SL, Link M]. Stereotactic radiosurgery of intracranial meningiomas. Neurosurg Clin N Am 2013; 24: 499-507. doi:10.1016/j.nec.2013.05.006

[23] Lee JY, Niranjan A, Mclnerney J et al. Stereotactic radiosurgery providing long-term tumor control of cavernous sinus meningiomas. J Neurosurg 2002; 97: 65-72. doi:10.3171/ jns.2002.97.1.0065

[24] Debus J, Wuendrich M, Pirzkall A et al. High efficacy of fractionated stereotactic radiotherapy of large base-of-skull meningiomas: long-term results. J Clin Oncol 2001; 19: 3547-3553. doi:10.1200/JCO.2001.19.15.3547

[25] Jenkinson MD, Weber DC, Haylock B] et al. Radiotherapy versus observation following surgical resection of atypical meningioma (the ROAM trial). Neuro-Oncology 2014; 16 : 1560-1561. doi:10.1093/neuonc/nou149

[26] El Shafie RA, Czech M, Kessel KA et al. Clinical outcome after particle therapy for meningiomas of the skull base: toxicity and local control in patients treated with active rasterscanning. Radiat Oncol 2018; 13: 54. doi:10.1186/s13014-0181002-5

[27] Coggins WS, Pham NK, Nguyen AV et al. A Systematic review of ion radiotherapy in maintaining local control regarding atypical and anaplastic meningiomas. World Neurosurg 2019; 132: 282-291. doi:10.1016/j. wneu.2019.08.149

[28] Lou E, Sumrall AL, Turner $S$ et al. Bevacizumab therapy for adults with recurrent/progressive meningioma: a retrospective series. J Neuro-Oncology 2012; 109: 63-70. doi:10.1007/s11060-012-0861-0

[29] Kaley TJ, Wen P, Schiff D et al. Phase II trial of sunitinib for recurrent and progressive atypical and anaplastic meningioma. Neuro-Oncology 2015; 17: 116-121. doi:10.1093/ neuonc/nou148
[30] Seystahl K, Stoecklein V, Schuller U et al. Somatostatin receptor-targeted radionuclide therapy for progressive meningioma: benefit linked to 68Ga-DOTATATE/-TOC uptake. Neuro-Oncology 2016; 18: 1538-1547. doi:10.1093/neuonc/now060

[31] Preusser M, Le RhunE, Soffietti R et al. Trabectedin for recurrent WHO grade II or III meningioma: A randomized phase Il study of the EORTC Brain Tumor Group (EORTC1320-BTG). J Clinl Oncol 2019; 37: no. 15_suppl. doi:10.1200/JCO.2019.37.15_suppl.2007

[32] Goldbrunner R, Minniti G, Preusser M et al. EANO guidelines for the diagnosis and treatment of meningiomas. Lancet Oncol 2016; 17: e383-391. doi:10.1016/S1470-2045(16) 30321-7

[33] Simpson D. The recurrence of intracranial meningiomas after surgical treatment. J Neurol Neurosurg Psychiat 1957; 20: 22-39. doi:10.1136/jnnp.20.1.22

[34] Mirimanoff RO, Dosoretz DE, Linggood RM et al. Meningioma: analysis of recurrence and progression following neurosurgical resection. J Neurosurg 1985; 62: 18-24. doi:10.3171/jns.1985.62.1.0018

[35] van Alkemade $\mathrm{H}$, de Leau M, Dieleman EM et al. Impaired survival and long-term neurological problems in benign meningioma. Neuro-Oncology 2012; 14: 658-666. doi:10.1093/neuonc/nos013

[36] Glaholm J, Bloom H], Crow JH. The role of radiotherapy in the management of intracranial meningiomas: the Royal Marsden Hospital experience with 186 patients. Int J Radiat Oncol Biol Phys 1990; 18: 755-761. doi:10.1016/03603016(90)90394-y

[37] Palma L, Celli P, Franco C et al. Long-term prognosis for atypical and malignant meningiomas: a study of 71 surgical cases. J Neurosurg 1997; 86: 793-800doi:10.3171/ jns.1997.86.5.0793

[38] Sahm F, Schrimpf D, Stichel D et al. DNA methylation-based classification and grading system for meningioma: a multicentre, retrospective analysis. Lancet Oncol 2017; 18: 682694. doi:10.1016/S1470-2045(17)30155-9

\section{Bibliografie}

Neurologie up2date 2020; 03: 343-356

DOI 10.1055/a-0965-2007

ISSN 2511-3453

(c) 2020. Thieme. All rights reserved. Georg Thieme Verlag KG Rüdigerstraße 14, 70469 Stuttgart, Germany 


\section{Punkte sammeln auf CME.thieme.de}

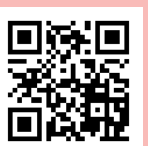

Diese Fortbildungseinheit ist in der Regel 12 Monate online für die Teilnahme verfügbar.

Den genauen Einsendeschluss finden Sie unter https://cme.thieme.de/CXDHLIN.

Sollten Sie Fragen zur Online-Teilnahme haben, finden Sie unter https://cme.thieme.de/hilfe

eine ausführliche Anleitung. Wir wünschen viel Erfolg beim Beantworten

der Fragen!

Unter https://eref.thieme.de/CXDHLIN oder über den QR-Code kommen Sie direkt zur Startseite des Wissenstests.

VNR 2760512020158724670

\section{Frage 1}

Welche Aussage zur WHO-Klassifikation von Meningeomen ist richtig?

A In der WHO-Klassifikation der Meningeome sind 6 verschiedene gutartige Meningeome ausgewiesen.

B Alle Meningeome werden dem WHO-Grad-I zugeordnet.

C Bei Meningeomen werden 15 verschiedene histologische Subtypen beschrieben.

D NF-2-Mutationen sind Teil der WHO-Klassifikation von Meningeomen.

E WHO-Grad-III-Meningeome sind gutartige Hirntumoren.

\section{Frage 2}

Nur eine der folgenden Aussagen zur Primärprävention von Meningeomen ist richtig. Welche?

A Meningeome werden durch UV-Strahlung verursacht.

B Hormonersatzpräparate spielen keine Rolle bei der Entstehung von Meningeomen.

C Untergewicht wird mit einem erhöhten Meningeomrisiko assoziiert.

D Für Meningeome kann eine familiäre Disposition bestehen

E Nikotinkarenz schützt vor Meningeomen.

\section{Frage 3}

Welches ist die häufigste Symptomatik, die zur Diagnose eines Meningeoms führt?
A Kopfschmerzen
B morgendliche Übelkeit
C fokal-neurologische Ausfälle
D symptomloser Zufallsbefund
E erstmaliger epileptischer Anfall

\section{Frage 4}

Diagnostisches Verfahren der Wahl bei Verdacht auf ein Meningeom ist ...
A die cCT.
B die MRT.
C das EEG.
D die Angiografie.
E die Liquoruntersuchung.

\section{Frage 5}

Was versteht man unter dem „Dural Tail Sign“?

A Das Tumorödem um ein Meningeom.

B Die homogene Kontrastmittelanreicherung eines Meningeoms.

C Verdickung der Pia mater im Randbereich des Meningeoms durch die Tumorinfiltration.

D Verdickung der Arachnoidea im Randbereich des Meningeoms durch die Tumorinfiltration.

E Verdickung der Dura im Randbereich des Meningeoms durch die Tumorinfiltration.

\section{Frage 6}

Was ist der Goldstandard zur Behandlung von Meningeomen?

A die operative Resektion

B die Strahlenchirurgie

C die primäre Chemotherapie

D eine Therapie mit Antiangiogenese-Hemmern

E eine Immuntherapie

\section{Frage 7}

Wann ist keine Bestrahlung bei Meningeomen indiziert?

A bei inkompletter Resektion eines WHO-Grad-III-Meningeoms

B bei inkomplett entfernten WHO-Grad-II-Meningeomen

C bei operativ nicht zugänglichen Meningeomen

D bei kompletter Resektion eines WHO-Grad-III-Meningeoms

E bei komplett entfernten WHO Grad-I-Meningeomen 


\section{Punkte sammeln auf CME.thieme.de}

Fortsetzung $\ldots$

\section{Frage 8}

Welche Systemtherapie kann bei Versagen der Lokaltherapien eingesetzt werden?
A Temozolomid
B Lomustin
C Bevacizumab
D Mifepriston
E Tamoxifen

\section{Frage 9}

Welches Medikament zur Behandlung von Meningeomen wurde kürzlich in einer multizentrischen randomisierten klinischen Studie untersucht?
A Methadon
B Metformin
C Trabectedin
D Idasanutlin
E Palbociclib

\section{Frage 10}

Welches Vorgehen wird bei der Nachsorge von Meningeomen empfohlen?
A 3-monatliche Verlaufskontrollen über 2 Jahre
B jährliche Verlaufskontrollen über 2 Jahre
C eine postoperative Verlaufskontrolle nach 3 Monaten und anschließend jährliche Verlaufskontrollen
D halbjährliche Verlaufskontrollen
E zweijährliche Verlaufskontrollen 